\title{
Guest Editorial: Robust and Secure Data Hiding Techniques for Telemedicine Applications
}

\author{
Amit Kumar Singh ${ }^{1} \cdot$ Basant Kumar $^{2}$. \\ Sanjay Kumar Singh ${ }^{3}$ - Mayank Dave ${ }^{4}$. \\ Vivek Kumar Singh ${ }^{5}$ - Pardeep Kumar ${ }^{1}$ • S. P. Ghrera ${ }^{1}$ • \\ Pradeep Kumar Gupta ${ }^{6}$ - Anand Mohan ${ }^{7}$
}

Recently, with the explosive growth of information and communication technologies, various new opportunities emerged for the creation and delivery of content in digital form which includes applications such as real time video and audio delivery, electronic advertising, digital libraries, broadcast monitoring, e-governance, e-commerce applications, copy control, media

Amit Kumar Singh

amit_245singh@yahoo.com

Basant Kumar

singhbasant@yahoo.com

Sanjay Kumar Singh

sks.cse@iitbhu.ac.in

Mayank Dave

mdave67@gmail.com

Vivek Kumar Singh

vivekks12@gmail.com

Pardeep Kumar

pardeepkumarkhokhar@gmail.com

S. P. Ghrera

sp.ghrera@juit.ac.in

Pradeep Kumar Gupta

pkgupta@ieee.org

Anand Mohan

profanandmohan@gmail.com

1 Department of Computer Science and Engineering, Jaypee University of Information Technology, Waknaghat, Solan, Himachal Pradesh, India 
identification and tracking, healthcare, E-Voting Systems, remote education, web publishing and digital cinema. One of the promising applications of digital image watermarking is in the field of secure medical data transmission for various telemedicine applications. However, these advantages have the consequent risks of data piracy and authenticity, which motivate towards development of new protection mechanisms. One such effort that has been attracting interest is based on digital watermarking techniques, which is a technique for embedding data (called a watermark) into digital multimedia cover objects in such a way that the watermark can be detected or extracted later to make an assertion about the authenticity and / or originality of the object. The aim of this special issue is to solicit the state-of-the-art data hiding techniques and technical solutions in the area of telemedicine applications.

This special issue has attracted 58 manuscripts and the submissions have been strictly reviewed by least three reviewers consisting of guest editors and external reviewers, with 23 high-quality articles accepted in the end. Below, we briefly summarize the highlights of each paper.

In Reversible data hiding in encrypted images based on multi-level encryption and block histogram modification (10.1007/s11042-016-4049-z). Yin et al. propose a reversible data hiding technique in encrypted images (RDH-EI). The smaller location map and block histogram modification (BHM) using a self-hidden peak pixels technique contribute to reduce the quantity of side information and improve the pure payload. Further, these techniques are combined with proposed multi-level encryption (MLE) method using Josephus traversal and stream cipher an integrated RDH-EI framework. The results demonstrate that the distortion of the decrypted images is smaller, lossless recovery was achieved in every evaluated case, and the payload is larger, which is better than other state-of-the-art research used for comparison.

In Hybrid NSCT domain multiple watermarking for medical images (10.1007/s11042-016-38851). Singh et al. presents a hybrid technique of multiple watermarking for medical images using nonsubsampled contourlet transform (NSCT), discrete cosine transform (DCT), multi-resolution singular value decomposition (MSVD) and Arnold transform. The method is evaluated with a different medical cover images and three different watermarks. Further, accuracy of the method is enhanced by using proper selection gain factor and NSCT sub-bands. The results demonstrate that the proposed method is suitable for recovery of multiple watermarks. Furthermore, robustness comparisons of the proposed technique show the superiority with other state-of-the-art algorithms.

In An efficient approach for face recognition in uncontrolled environment (10.1007/s11042-0163976-z). Agrawal and Singh develop a framework of face recognition system that outperforms in unconstrained environments. The framework works on features based method that extracts facial landmarks from images. After quality check the patch experts are generated and used to model the

2 Department of Electronics Engineering, Motilal Nehru National Institute of Technology, Allahabad, Uttar Pradesh, India

3 Department of Computer Science \& Engineering, Indian Institute of Technology (Banaras Hindu University), Varanasi, India

4 Department of Computer Engineering, National Institute of Technology, Kurukshetra, Haryana, India

5 Department of Computer Science, Banaras Hindu University, Varanasi, Uttar Pradesh, India

6 University of Pretoria, Pretoria, South Africa

7 Department of Electronics Engineering, Indian Institute of Technology (Banaras Hindu University), Varanasi, Uttar Pradesh, India 
appearance of landmarks of interests. The effect of discriminatory features is further enhanced by assigning weights to them that are to be set to the ratio of the interclass variance to the intra-class variance. The results show that the proposed framework achieves better recognition accuracy in comparison to other state-of-the-art algorithms on publically available datasets.

In Data hiding in AMBTC images using quantization level modification and perturbation technique (10.1007/s11042-016-3977-y). Hong et al. present $a$ data hiding technique for absolute moment block truncation coding (AMBTC) compressed image using quantization level modification. Blocks of AMBTC-compressed image are classified into two categories, namely smooth and complex, according to a predefined threshold. For smooth blocks, the bitmap is replaced by secret data for data embedment. Meanwhile, the corresponding quantization levels are modified to achieve a minimum distortion. Results indicate that the modified quantization levels can be further perturbed for carrying two additional bits, if a larger payload is required. If the blocks are complex, one data bit can be embedded with no distortion by swapping the values of the two quantization levels together with bitmap flipping. In addition, a suppress threshold mechanism is used to prevent from the application of the perturbation technique at low payload to maintain the image quality. The proposed method minimizes the distortion of each stego block while ensuring high payload, thus the embedding efficiency is enhanced. Experimental evaluations demonstrate the improvement of the proposed method compared with other related state-of-art research.

In Joint JPEG Compression and Detection Resistant Performance Enhancement for Adaptive Steganography Using Feature Regions Selection (10.1007/s11042-016-3914-0). Zhang et al. focus a JPEG compression and detection resistant adaptive steganography algorithm using feature regions. Based on the proposed feature region extraction and selection algorithms, the embedding domain robust to JPEG compression and containing less embedding distortion can be obtained. Further, combined with error correct coding and SyndromeTrellis codes (STCs), the messages are embedded into the cover images with minimum embedding distortion, and can be extracted with high accuracy after JPEG compression, hence, the JPEG compression and detection resistant performance are enhanced at the same time. Experimental evaluations demonstrate the improvement of the proposed method compared with current J-UNIWARD steganography.

In A blind medical image watermarking: DWT-SVD based robust and secure approach for telemedicine applications (10.1007/s11042-016-3928-7). Thakkar and Srivastava present a blind image watermarking technique for telemedicine application using discrete wavelet transform (DWT) and singular value decomposition (SVD). Two watermark contents in the form of image and electronic patient record (EPR) watermark are embedded into the SVD blocks of selected wavelet sub-band of the medical cover image. The watermark contents are embedded in such a way that blind extraction of the contents becomes possible at the receiving end. Instead of directly embedding of EPR watermark, Hamming error correcting code is applied on it to get encoded watermark which is embedded into the image. The method is evaluated and found to be robust under different signal processing attacks including checkmark attacks. The Experimental analysis shows that the proposed work achieves better robustness in comparison to other state-of-the-art algorithms at acceptable visual quality of the watermarked image. Moreover, proposed technique is also appropriate for color image watermarking.

In A multimodal biometric watermarking system for digital images in redundant discrete wavelet transform (10.1007/s11042-016-4048-0). Singh et al. develop a multimodal biometric watermarking system using redundant discrete wavelet transform (RDWT). Two biometric traits iris and facial features are embedded independently into the sub-bands of the RDWT of cover image taking advantage of its translation invariant property and sufficient embedding capacity. 
The ownership verification accuracy of the proposed system is evaluated based on the individual biometric traits as well as the fused trait. The accuracy is enhanced while using the fused score for evaluation. The security of the method is strengthened with usage of non-linear chaotic maps, randomization through Hessenberg decomposition, Arnold scrambling and multiple secret keys. The robustness of the method is tested for various important attacks and the verification accuracy is evaluated based on false acceptance rate, false rejection rate, area under curve and equal error rate to validate the efficacy of the proposed method.

In Robust covert communication using high capacity watermarking (10.1007/s11042-016-3978$\mathrm{x})$. Bhatnagar presents a high-capacity watermarking technique in which watermarkable pixels are selected from the cover image based on the census transform and hamming distance followed by the embedding which is done by proposed spectral decompositions. Finally, a reliable watermark extraction technique is developed which is free from the false-positive detection problem of singular values. The experimental evaluations demonstrate that the proposed technique is expeditiously able to withstand a various kind of extreme attacks and highly suitable for covert communications.

In Statistical textural feature and deformable model based brain tumor segmentation and volume estimation (10.1007/s11042-016-3979-9). Banday and Mir present a semi-automatic technique that yields appropriate segmented regions from MR brain images. The segmentation technique here utilizes fusion of information beyond human perception from MR images to develop a fused feature map. The information beyond human perception includes second order derivatives that are determined from an image. This obtained feature map acts as a stopping function for the initialized curve in the framework of an active contour model to obtain a well segmented region of interest. The segmentation is carried out in all the slices of a particular dataset with initialization of the active contour required only on the first slice which makes this method fast. The obtained segmentation results are compared with ground truth segmentation results obtained from experts manually using Jackard's Co-efficient of Similarity and Overlap index. The efficacy of this volume estimation technique is illustrated with comparison to mostly used $\mathrm{ABC} / 2$ method and cavalieri method. The results obtained on various case studies like Craniophryngioma, High grade Glioma and Microadenoma show a good efficacy of the method.

In Digital watermark extraction in wavelet domain using hidden Markov model (10.1007 /s11042-016-3975-0). Amini et al. propose a watermark decoder using the vector-based hidden Markov model (HMM) in the wavelet domain. The watermark decoder is designed based on the maximum likelihood criterion. Closed-form theoretical expression for the watermark decoder is derived. The performance of the proposed decoder in terms of bit error rate values is assessed using a number of test images. The results indicate that the proposed decoder is robust for different kind of attacks and superior to other decoders in terms of providing a lower bit error rate.

In A new reversible and high capacity data hiding technique for e-healthcare applications (10.1007/s11042-016-4196-2). Parah et al. propose a high capacity and reversible data hiding technique capable of tamper detection and localization of medical images. The cover image is divided into non-overlapping blocks. In every block pixels are classified into two types: Seed pixels and non-seed pixels. The EPR is embedded only in non-seed pixels while as no embedding is carried out in seed pixels to facilitate reversibility. A fragile watermark coupled with block checksum is embedded in addition to EPR for detecting any tamper to the patient data during its transit from sender to receiver. Embedding has been carried out using intermediate significant bit substitution (ISBS) to prevent the technique from LSB removal/replacement attack. The technique is evaluated for perceptual imperceptibility and content authentication by subjecting it to various image processing and geometric attacks. Experimental results reveal that the proposed system is capable of providing high quality watermarked images for fairly high payload while 
maintaining reversibility. Further, the proposed technique is able to detect tamper for all the image processing and geometric attacks carried out on it. A comparison of the observed results with that of some state-of-art algorithms show that the method performs better and as such is an ideal candidate for content authentication of EPR in a typical healthcare system.

In Data hiding using pseudo magic squares for embedding high payload in digital images (10.1007/s11042-016-3974-1). Ranjani propose a data hiding technique using pseudo magic square pattern. The pattern is generated using the Knight's move algorithm, whereas diamond encoding (DE) and adaptive pixel pair matching (APPM) embedding schemes adapts rhombic shaped and non-uniform pattern respectively. The momentous feature of the proposed embedding scheme is its ability to generate several pseudo magic squares for a given embedding parameter. The performance analysis in the experimental results reveal that the proposed algorithm not only provides increased payload with less distortion but also increases the security of the embedding processing by allowing the application to employ one among the several square patterns for every secure communication.

In A light weight and secure video conferencing scheme utilizing public network (10.1007 /s11042-016-3973-2). Meenpal present a selective encryption technique applied on the quantized non-zero DCT coefficients of the macro-blocks belonging to I-frame of the JPEG/MPEG family such that the resultant image/video is completely obscure and is suitable for high end security applications. Also there is no reduction in the performance of compression algorithms applied later in the standard JPEG/MPEG pipeline. Experiments show that the encrypted image/video file is almost of the same size as that of un-encrypted version. Thus the scheme is suitable for telemedicine as well as other important applications.

In Iterative quadtree decomposition based automatic selection of the seed point for ultrasound breast tumor images (10.1007/s11042-016-3761-z). Fan et al. explore an automatic method for finding the seed point inside the tumor. Two criteria combining iterative quadtree decomposition (QTD) and the gray characteristics of the lesion are thus designed to locate the seed point. One is to seek the biggest homogenous region and the other is to select the seed region where the seed point is found. Furthermore, this study validates the proposed algorithm through various ultrasonic breast tumor images. As the quantitative experiment results show, the proposed method can automatically find the seed point inside the tumor with satisfactory performance in term of sensitivity and accuracy rate.

In Dual domain robust watermarking scheme using random DFRFT and least significant bit technique (10.1007/s11042-016-4095-6). Sharma et al. develop a robust and non-blind image watermarking method that uses multiple-parameter discrete fractional Fourier transform (MPDFRFT) with random DFRFT and least significant bit (LSB) technique. The simulation results validate that the embedding method has better performance in terms of robustness along with perceptibility and security over contemporary existing techniques.

In Region-based hybrid medical image watermarking for secure telemedicine applications (10.1007/s11042-016-3913-1). Singh and Maheshkar present a region based hybrid medical image watermarking (MIW) technique using fusion of IWT and SVD. Initially, the cover medical image is partitioned into region of interest (ROI) and the region of non interest (RONI). To detect and localize ROI tampering with high accuracy pixel wise positional and relational bits are calculated. Two original LSBs of each ROI pixel are replaced by their corresponding positional and relational bits. Original LSBs of ROI pixels are concatenated and embedded in RONI for ROI recovery in the case of tampering. Multiple watermarks i.e. EPR, hospitals logo and LSBs of ROI are embedded simultaneously as a robust watermark in RONI using IWT-SVD hybrid transform. The proposed technique is blind and free from false positive detection. Various experiments is carried out on different medical imaging modalities to evaluate the performance of the proposed 
technique in terms of imperceptibility, robustness, tamper detection, localization, recovery and computation time. ROI tampering is detected and recovered with high accuracy.

In Unified entropy-based sorting for reversible data hiding (10.1007/s11042-016-3989-7). Xu et al. propose an entropy-based sorting (EBS) method for reversible data hiding, which uses entropy measurement to characterize local context complexity for each image pixel. Furthermore, by extending the EBS technique to the two-dimensional case, it shows generalized abilities for multidimensional RDH scenarios. Additionally, a new gradient-based tracking and weighting (GBTW) pixel prediction method is introduced to be combined with the EBS technique. Experimental results apparently indicate that the proposed method outperforms the previous state-of-arts counterparts significantly in terms of both the prediction accuracy and the overall embedding performance.

In A robust blind hybrid image watermarking scheme in RDWT-DCT domain using Arnold scrambling (10.1007/s11042-016-3902-4). Roy and Pal propose a robust and blind image watermarking technique using Redundant Discrete Wavelet Transform (RDWT), Discrete Cosine Transform (DCT), Arnold scrambling and error correction repetition code. Experimental results show that robustness is achieved by recovering satisfactory watermark data from the reconstructed cover image after applying common geometric transformation attacks, common enhancement technique attacks, JPEG compression and Checkmark attacks.

In Accelerating Compute Intensive Medical Imaging Segmentation Algorithms Using GPUs (10.1007/s11042-016-3884-2). Alsmirat et al. focus on Fuzzy C-Means based segmentation algorithms and accelerates the execution time of these algorithms using Graphics Process Unit (GPU) capabilities. In this research, authors are studied pure GPU and hybrid CPU-GPU implementations with CUDA. The result outcomes show that the hybrid CPU-GPU improves the execution time much better than that of the pure GPU implementations.

In XOR based Continuous-tone Multi Secret Sharing for Store-and-forward Telemedicine (10.1007/s11042-016-4012-z). Shivani et al. present XOR based Continuous-tone Multi Secret Sharing scheme suitable for store-and-forward telemedicine application. It also eliminates basic security constraints of visual cryptography (VC) like pixel expansion in shares/recovered secret images, random pattern of shares, explicit codebook requirement, lossy recovery of secret and limitation on number of secret and shares. Proposed technique is ' $n$ ' out of ' $n$ ' multi secret sharing scheme which is able to transmit ' $n$ ' secret images simultaneously. All secrets could be revealed only after some computations with all ' $n$ ' shares and one master share. Master share has been created with the secret key at encoding phase and it can be regenerated at the time of decoding using same secret key. Proposed technique not only preserves all basic characteristics of traditional VC but also increases the capacity of secret image sharing. From the experiments we found that irrespective of visible contents of the shares, the probability of getting back the pixel values of respective original secret images at the receiver end is very high.

In Mobile-cloud assisted framework for selective encryption of medical images with steganography for resource-constrained devices (10.1007/s11042-016-3811-6). Sajjad et al. address the problem of outsourcing the selective encryption of a medical image to cloud by resource-constrained devices, without revealing the cover image to cloud using steganography. In the proposed technique, the region of interest of the medical image is first detected using a visual saliency model. The detected important data is then embedded in a host image, producing a stego image which is outsourced to cloud for encryption. The cloud which has powerful resources, encrypts the image and sent back the encrypted marked image to the client. The client can then extract the selectively encrypted region of interest and can combine it with the region of non-interest to form a selectively encrypted image, which can be sent to medical specialists and healthcare centers. Experimental results and analysis 
validate the effectiveness of the proposed framework in terms of security, image quality, and computational complexity and verify its applicability in remote patient monitoring centers.

In (Two-dimensional) ${ }^{2}$ whitening reconstruction for newborn recognition (10.1007/s11042016-3731-5). Singh and Om propose a (Two-dimensional) ${ }^{2}$ whitening reconstruction $\left(\mathrm{T}^{2} \mathrm{WR}\right)$ pre-processing step along with Principal Component Analysis (PCA) algorithm which is a stateof-the-art technique to overcome the challenge of recognizing faces under different lightening conditions. This technique is applied to newborn face database which is a rarely accessible database. The technique is compared with state-of-the-art Two-dimensional whitening reconstruction (TWR) pre-processing algorithm and indicates better performance of $\mathrm{T}^{2} \mathrm{WR}$ over TWR. The histograms plotted for both these algorithms show that $\mathrm{T}^{2} \mathrm{WR}$ makes for a smoother frequency distribution than TWR. The proposed method proved to be comparatively more robust in identification of newborns in variety of illumination, expression change and occlusive image.

In Double expanding robust image watermarking based on Spread Spectrum technique and BCH coding (10.1007/s11042-016-3734-2). Soleymani and Taherinia develop an algorithm for blind image watermarking which has a high robustness against common image processing attacks. In this research, a small watermark of size is double expanded into multi larger meaningful bits with applying $\mathrm{BCH}$ error correction code and spread spectrum technique in order to reduce errors in extraction phase. Consequently this method allocates smaller gain factors to smooth blocks and larger gain factors to texture and rough blocks. The simulation results show that better performance in terms of robustness and visual quality over contemporary existing techniques.

These 23 selected contributions basically can reflect the new achievements in the telemedicine applications and we hope they can provide a solid foundation for future new approaches and applications. Finally, we would like to thank all authors for their contributions, the reviewers for reviewing these high quality papers, and Editor-in-Chief, Prof. Borko Furht, for his support and guidance throughout the process.

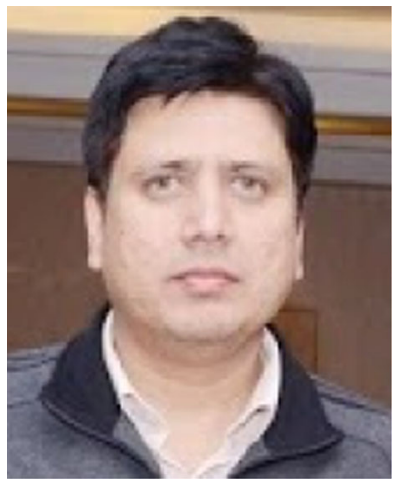

Dr. Amit Kumar Singh is currently working as Assistant Professor (Senior Grade) in the Department of Computer Science \& Engineering at Jaypee University of Information Technology (JUIT) Waknaghat, Solan, Himachal Pradesh-India since April 2008. He has completed his PhD degree from the Department of Computer Engineering, NIT Kurukshetra, Haryana in 2015. Recently, Dr. Singh appointed as Associate Editor of IEEE Access and Multimedia Tools and Applications (MTAP), Springer. He has presented and published over 45 research papers in reputed journals and various national and international conferences. His important research contributions includes to develop watermarking methods that offer a good trade-off between major parameters i.e. perceptual quality, robustness, embedding capacity and the security of the watermark embedding into the cover digital images. His research interests include Data Hiding, Biometrics \& Cryptography. 


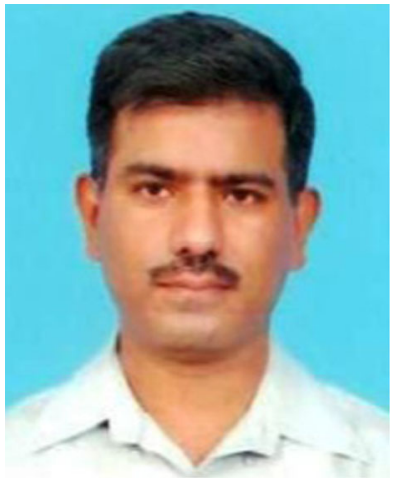

Dr. Basant Kumar is currently working as Assistant Professor in Department of Electronics and Communication Engineering, Motilal Nehru National Institute of Technology, Allahabad. He has more than 13 years of teaching and research experience. He obtained his B.Tech. degree in Electronics and Instrumentation Engineering from Bundelkhand Institute of Engineering and Technology, Jhansi, Uttar Pradesh, and M.E. degree in Communication Engineering from Birla Institute of Technology and Science, Pilani, in 1999 and 2002 respectively. He received Ph.D. in Electronics Engineering from Indian Institute of Technology, Banaras Hindu University, Varanasi, India (IIT-BHU) in 2011. His area of research includes telemedicine, data compression, data hiding, multimedia communication and medical image processing. He has published more than 30 research papers in reputed international journals/conferences.

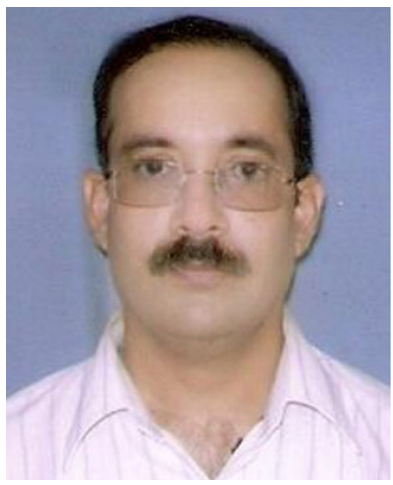

Dr. Sanjay Kumar Singh is currently working as Associated Professor at the Department of Computer Science and Engineering, IIT BHU, Varanasi. He has completed his B. Tech. in Computer Engg., M. Tech. in Computer Applications and Ph.D. in Computer Science and Engineering. He is a Certified Novell Engineer (CNE) from Novell Netware, USA and a Certified Novell Administrator (CNA) from Novell Netware, USA. He is a member of LIMSTE, IEE, International Association of Engineers and ISCE. His research areas include Biometrics, Computer Vision, Image Processing, Video Processing, Pattern Recognition and Artificial Intelligence. He has over 50 national and international journal publications, book chapters and conference papers. He is also a Guest Editorial Board Member of Multimedia Application and Tools, Springer, and the EURASIP Journal of Image and Vision Processing (Springer). He is a member of the Computer Society and the Association for Computing Machinery. 


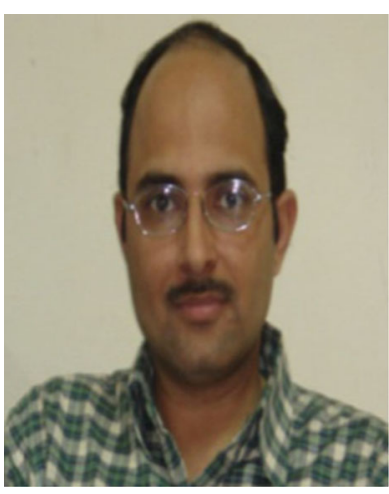

Prof. Mayank Dave working as Professor in the Department of Computer Engineering, NIT, Kurukshetra, Haryana-India. Dr. Dave obtained his Ph.D. (Computer Engineering) and M. Tech (Computer Engineering) from IIT Roorkee and he has 27 years rich experience of serving both academia and industry in various capacities. His research interest includes System and Network Security, Cyber Security, Cloud Security, Key Management, Wireless Sensor Networks, Underwater Sensor Networks, Semantic Web and Mobile Computing

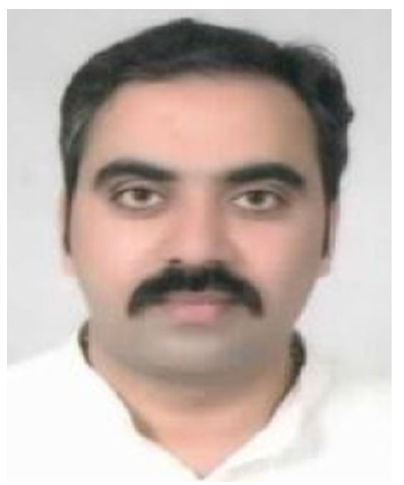

Dr. Vivek Kumar Singh obtained Bachelors' degree in Computer Science and Mathematics from the University of Allahabad in 1999 and Masters' degree in Computer Science (with First Rank and Honours) from the University of Allahabad in 2001. He has obtained D. Phil. degree in Science from the University of Allahabad in March 2010. The area of his Doctoral research work was Collective Intelligence. His research interests includes Artificial Intelligence, Complex Adaptive Systems, Social Computing and Web Technology 


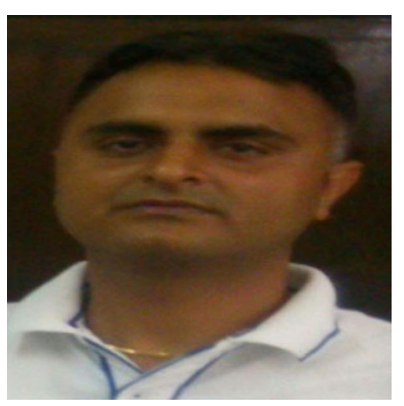

Dr. Pardeep Kumar is currently working as Assistant Professor (Senior Grade) in the Department of Computer Science \& Engineering at Jaypee University of Information Technology (JUIT), Wakanaghat and he has 10 years of extensive experience in Academics. Prior to joining Jaypee Group, he has associated with Mody University of Technology \& Science (Formerly known as Mody Institute of Technology \& Science) Laxmangarh, Sikar, Rajasthan. He has completed his Ph.D. (Computer Science and Engineering, Nov. 2012) from Uttarakhand Technical University, Dehradun. Dr. Kumar is also serving as Professional Member of ACM (Association for Computing Machinery), Life Member of IAENG (International Association of Engineers) and IAENG society of computer science and society of DataMining. Dr. Kumar has published around 22 papers in peer reviewed Journals and Conferences of National and International repute.

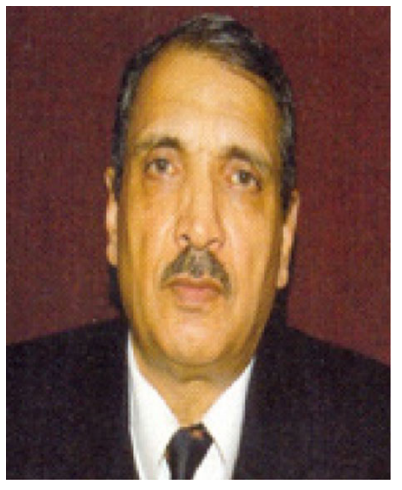

Prof. Satya Prakash Ghrera completed 34 years of service in Corps of Electronics and Mechanical Engineers of the Indian Army, he joined Jaypee Institute of Engineering and Technology in Jan. 2006 as Associate Professor in the Department of Computer Science and Engineering. With effect from Sep. 2006, he has taken over responsibilities of HOD (Computer Science Engg. and IT) at Jaypee University of Information Technology Waknaghat, Distt. Solan HP. His research interests include Information Security \& Cryptography. 


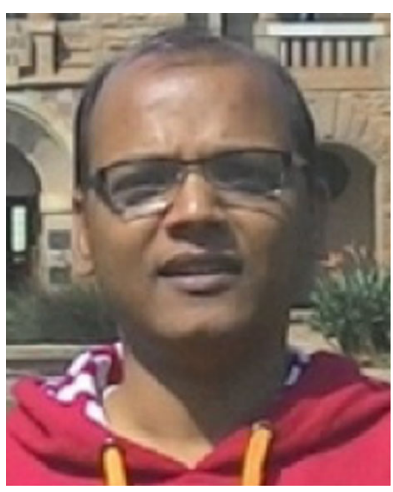

Dr. P. K. Gupta is Post-Doctorate from University of Pretoria, South Africa in the Department of Electrical, Electronic and Computer Engineering. He is working as a Assistant Professor (Sr. Grade) at Jaypee University of Information Technology, Solan, Himachal Pradesh, India. He has 15 years of extensive experience in IT industry and Academics in India and abroad. Dr. Gupta was the recipient of NRF South Africa grant. He has received award from Computer Society of India for maximum publication during year 2015-16. Dr. Gupta is associate editor of IEEE Access and also serving as a reviewer and editorial board member of many reputed international journals and conferences.

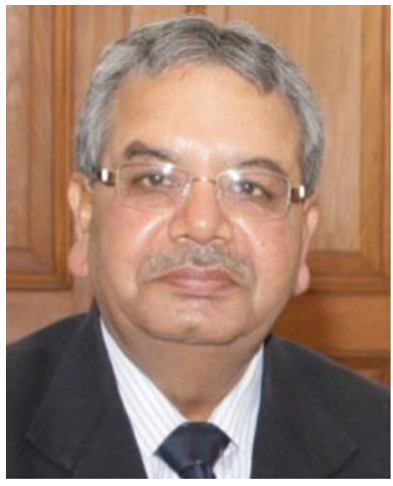

Prof. Anand Mohan is a Professor of Electronics Engineering at Indian Institute of Technology, Banaras Hindu University where he has held as several important administrative positions namely Member of Executive Council, Head of the Department of Electronics Engineering, Coordinator, Centre for Research in Microprocessor Applications (established by MHRD), and In charge, University Science Instrumentation Centre. Prof. Mohan has 35 years rich experience of serving both academia and industry in various capacities. Prof. Mohan obtained Ph. D., PG, and UG degrees in Electronics Engineering from Banaras Hindu University in 1994, 1977, and 1973 respectively. He has made notable contributions to the academic and research development in Electronics Engineering at Banaras Hindu University by creating dedicated research groups of eminent academic experts from the country and abroad. He conducted high quality research in the emerging areas like fault tolerant / survivable system design, information security, and embedded systems. 